\title{
Management of Cardiomyopathy in Pediatric Cardiology Unit at Assiut University Children Hospital (Clinical Audit)
}

\author{
SAFIEA A. EL-DEEB, M.D.; DUAA M. RAAFAT, M.D. and AML H.H. AHMED, M.Sc. \\ The Department of Cardiology, Pediatric Cardiology Unit, Assiut University Children Hospital, Faculty of Medicine, \\ Assiut University, Assiut, Egypt
}

\begin{abstract}
Background: This study aims to assess how much the staff in the Pediatric Cardiology Unit are sticking to the agreed upon unit's protocol in the management of children admitted with Dilated Cardiomyopathy (DCM). It is aiming also to compare the Cardiology Unit's agreed upon protocol to one of the international guidelines.
\end{abstract}

Methods: This audit was conducted on 25 children with Dilated Cardiomyopathy (DCM) admitted to Pediatric Cardiology Unit at Assiut University Children Hospital in the period between May 2015 and May 2016. The study included 20 males \& 5 females. Their ages ranged from 3 months to 16 years.

Results: The prevalence of male sex was $80 \%$ and $20 \%$ of cases were females. Cases $<3$ years constituted $40 \%$ of cases. In $60 \%$ of cases the age was older than $3 y$ up to 16 years of age; the number of cases presenting as first admission was $68 \%$ and in $32 \%$ of cases the admission was recurrent; carvidelol was used in $32 \%$ of children with DCM and in the rest of cases $(68 \%)$ carvidelol was not used; amiodarone was used in 2 cases ( $8 \%$ of the cases): In one case it was used alone, and in the second case it was used in combination with propranolol.

The unit's protocol was followed in $100 \%$ of cases by staff members of the Cardiology Unit at Assiut University Children Hospital.

Regarding the difference between unit's protocol and Canadian guidelines: There were three main differences. The 1st one was the absence in the unit's protocol of taking family history of cases with DCM. This was emphasized in the Canadian guidelines. The 2 nd difference was that in the unit's protocol amiodarone was the 1 st drug of choice in cases of DCM with arrhythmia. The ${ }^{3 r d}$ difference was the use of Lcarnitine and aspirin regularly initially and for follow-up in all cases with DCM in the unit's protocol. This point was not mentioned in the Canadian guidelines.

Conclusion: Due to lack of genetic studies performed by Assiut University Children Hospital in cases with DCM, taking family history of cardiac diseases has been omitted by

Correspondence to: Dr. Safiea A. El-Deeb, The Department of Cardiology, Pediatric Cardiology Unit, Assiut University Children Hospital, Faculty of Medicine, Assiut University, Assiut, Egypt the staff of the unit. Taking family history of cardiac diseases will be emphasized in future researches.

This is because idiopathic DCM is now thought to have genetic basis in many cases. Furthermore, besides emphasizing taking a family history this study will recommend family cardiac screening by echocardiography to be done for all family members or at least for both parents and young sibling.

Key Words: Cardiomyopathy - Pediatric Cardiology Unit's protocol-Canadian protocol-Assiut University Children Hospital - Audit.

\section{Introduction}

CARDIOMYOPATHIES are defined as diseases of the myocardium, which cause cardiac dysfunction with heart failure, arrhythmia, and even sudden death. Cardiomyopathies represent a major cause of morbidity and mortality in children and are a frequent indication for cardiac transplantation.

Cardiomyopathies are classified into five types based on anatomic and functional features; hypertrophic, dilated, restrictive, arrhythmogenic Right Ventricular (RV) and Left Ventricular (LV) noncompaction cardiomyopathies.

1- In hypertrophic Cardiomyopathy (HCM), there is massive ventricular hypertrophy with a smaller than normal ventricular cavity. Contractile function of the ventricle is enhanced, but ventricular filling is impaired by relaxation abnormalities.

2- Dilated Cardiomyopathy (DCM) is characterized by decreased contractile function of the ventricle associated with ventricular dilatation. The left atrium may also be enlarged. The end-diastolic and end-systolic dimensions of the left ventricle are increased, with a markedly reduced fractional shortening and ejection fraction of the LV.

3- Restrictive cardiomyopathy denotes a restriction of diastolic filling of the ventricles. Contractile 
function of the ventricle may be normal, but there is marked dilatation of both atria.

4- Arrhythmogenic cardiomyopathy denotes selective RV enlargement and often systolic bulging or areas of akinesia or dyskinesia.

5- Noncompaction cardiomyopathy, also known as LV noncompaction, LV hypertrabeculation, or spongy myocardium. The characteristic echocardiography findings are segmental thickening of the LV wall consisting of two layers with a thin, compacted epicardial layer and an extremely thickened noncompacted endocardial

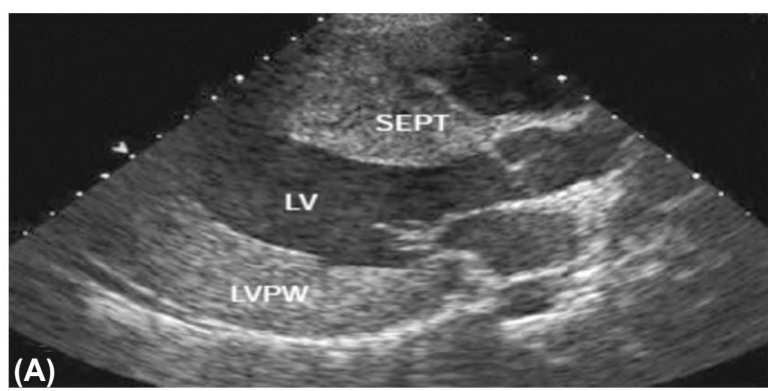

Fig. (1): Echocardiography showing hypertrophic cardiomyopathy.

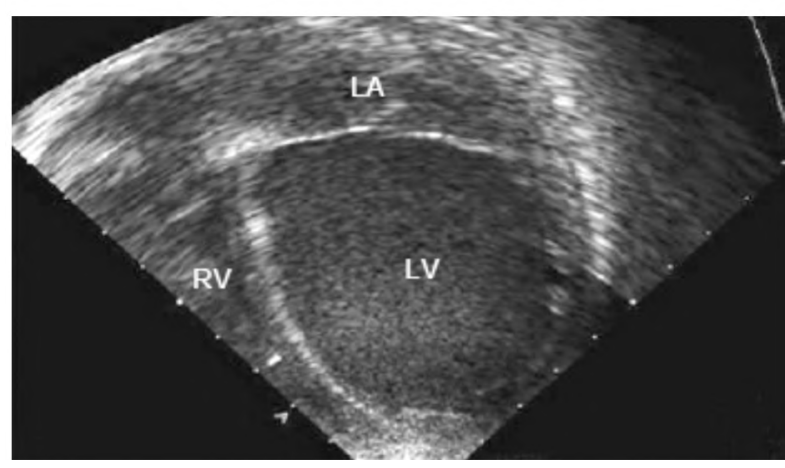

Fig. (2): Echocardiography showing dilated cardiomyopathy.

\section{Patients and Methods}

Study design:

This is an audit.

\section{Setting:}

The study was conducted in Pediatric Cardiology Unit at Assiut University Children Hospital from May 2015 to May 2016.

Inclusion criteria: All cases of dilated cardiomyopathy admitted to Pediatric Cardiology Unit at Assiut University Children Hospital during 1 year of study.

Exclusion criteria: All cases with known cause of heart failure due to congenital heart disease, acquired (e.g. RH, hypertensive, etc.), heart failure layer with prominent trabeculations and deep recesses. The LV is uniformly affected, resulting in systolic and diastolic dysfunction and clinical heart failure.

\section{Aim of work:}

The aim of this work is to assess how much the staff in the Pediatric Cardiology Unit are sticking to the agreed upon unit's protocol in the management of children admitted with Dilated Cardiomyopathy (DCM). And also to compare the Cardiology Unit's agreed upon protocol to one of the international guidelines.
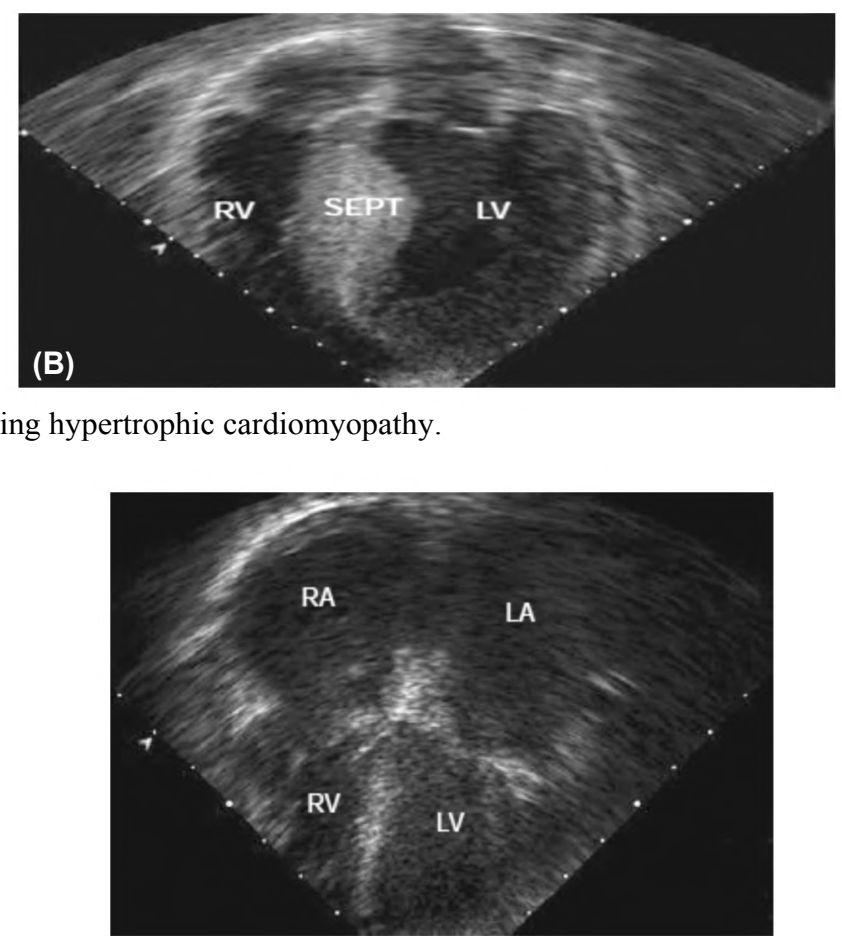

Fig. (3): Echocardiography showing restrictive cardiomyopathy.

with unknown cause or due to cor pulmonale and other types of cardiomyopathy.

\section{Population of study:}

25 children participated in the study in the period between May 2015 and May 2016.

All patients were subjected to the following:

A- History: Including personal history, history suggestive of congestive heart failure (shortness of breath, edema), history of cough or chest infection (wheezes, sputum, haemoptesis), history of palpitations.

$B$ - Physical examination: Including vital signs: pulse (rate, rhythm), blood pressure, temperature and respiratory rate, general examination, chest 
and heart examination, as well as manifestations of heart failure or thromboembolic manifestation and/or dysrhythmia with syncope or shock.

\section{C-Investigations:}

\section{I- Chest X-ray. \\ II- ECG. \\ III- ECHO. \\ IV- LDH \& CPK.}

Table (1): The unit's protocol.

$\begin{gathered}\text { Assiut Children Hospital Cardiology Unit Protocol } \\ \text { for management of dilated cardiomyopathy }\end{gathered}$
Name:
History:
1- A- Previous admission:
B- History suggestive of congestive heart failure (shortness of
breath, edema).
2- History of cough, chest infection (wheezes, sputum, haemoptesis).
3- History of palpitations.
Examination:
Vital signs: Pulse (rate, rhythm), blood pressure, temperature \&
respiratory rate.

\section{General examination:}

Chest \& heart examination:

Abdominal examination: Palpation of the liver, percussion for ascites.

CNS \& thromboembolic manifestations.

Investigations:

1- Chest X-ray.

2- ECG.

3- $\mathrm{ECHO}$

4- $\mathrm{LDH}, \mathrm{CPK}$

Tretment:

1- Furesmide I.V. 1-2mg/kg I.V. then $1-2 \mathrm{mg} / \mathrm{kg} /$ day orally.

2- Spironolactone $1-2 \mathrm{mg} / \mathrm{kg} /$ day orally in divided doses (tds).

3- Digoxin I.V. given only in maintenance dose $0.01 \mathrm{mg} / \mathrm{kg}$ in 2 equal doses.

4- Captopril (0.5-5mg/kg/day) in 2-4 equal doses orally.

5- A- (Amiodarone) 5mg/kg I.V. over 1 hour \& 5-15 mcg/ $\mathrm{kg} / \mathrm{min}$.

B- (Propranolol) $0.1-1 \mathrm{mg} / \mathrm{kg} /$ dose slowly I.V. \& repeat every hour if amiodarone was ineffective.

6- L-Carnitine (50-100mg/kg/day) orally in 2 equal doses continuously thereafter for life.

7- Aspirin (5-7mg/kg/day) orally for all patients and continuously thereafter.

8- Carvidelol (given orally in doe $0.1 \mathrm{mg} / \mathrm{kg} /$ day titrated up to $0.4 \mathrm{mg} / \mathrm{kg} /$ day) used in severe cases not responding to the routine use of Furesmide, Aspirin, Digoxin and Captopril.
Table (2): The unit's protocol checklist.

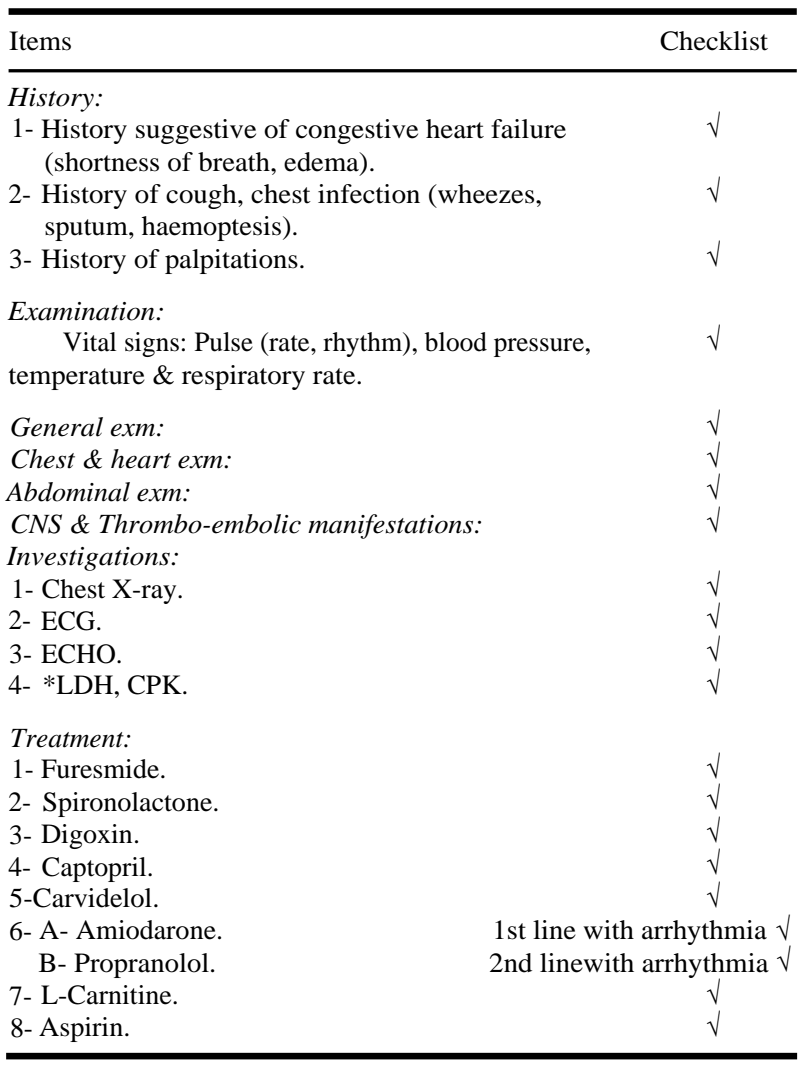

Table (3): The unit's protocol as compared and contrasted to Canadian Guidelines.

\begin{tabular}{|c|c|c|}
\hline Items & $\begin{array}{l}\text { Unit's } \\
\text { protocol }\end{array}$ & $\begin{array}{l}\text { Canadian } \\
\text { Guidelines }\end{array}$ \\
\hline \multicolumn{3}{|l|}{ History: } \\
\hline $\begin{array}{l}\text { 1- History suggestive of congestive heart } \\
\text { failure (shortness of breath, edema). }\end{array}$ & $\sqrt{ }$ & $\sqrt{ }$ \\
\hline $\begin{array}{l}\text { 2- History of cough, chest infection } \\
\text { (wheezes, sputum, haemoptesis). }\end{array}$ & $\sqrt{ }$ & $\sqrt{ }$ \\
\hline 3- History of palpitations. & $\sqrt{ }$ & $\sqrt{ }$ \\
\hline 4- Family history: Screening of the family. & $\mathrm{X}$ & $\sqrt{ }$ \\
\hline \multicolumn{3}{|l|}{ Examination: } \\
\hline $\begin{array}{l}\text { Vital signs: pulse (rate, rhythm), blood } \\
\text { pressure, temperature \& respiratory rate. }\end{array}$ & $\sqrt{ }$ & $\sqrt{ }$ \\
\hline General exm: & $\sqrt{ }$ & $\sqrt{ }$ \\
\hline Chest \& heart exm: & $\sqrt{ }$ & $\sqrt{ }$ \\
\hline Abdominal exm: & $\sqrt{ }$ & $\sqrt{ }$ \\
\hline CNS \& Thrombo-embolic manifestations: & $\sqrt{ }$ & $\sqrt{ }$ \\
\hline \multicolumn{3}{|l|}{ Investigations: } \\
\hline 1- Chest X-ray. & $\sqrt{ }$ & $\sqrt{ }$ \\
\hline 2- ECG. & $\sqrt{ }$ & $\sqrt{ }$ \\
\hline 3- ECHO. & $\sqrt{ }$ & $\sqrt{ }$ \\
\hline 4- LDH, CPK. & $\sqrt{ }$ & $\mathrm{X}$ \\
\hline \multicolumn{3}{|l|}{ Treatment: } \\
\hline 1- Furesmide. & $\sqrt{ }$ & $\sqrt{ }$ \\
\hline 2- Spironolactone. & $\sqrt{ }$ & $\sqrt{ }$ \\
\hline 3- Digoxin. & $\sqrt{ }$ & $\sqrt{ }$ \\
\hline 4- Captopril. & $\sqrt{ }$ & $\sqrt{ }$ \\
\hline 5- Carvidelol. & $\sqrt{ }$ & $X$ \\
\hline 6- A- Amiodarone. & rrhythmia $\sqrt{ }$ & $X$ \\
\hline B- Propranolol. & rrhythmia $\sqrt{ }$ & $\mathrm{X}$ \\
\hline $\begin{array}{l}\text { 7- L-Carnitine. } \\
\text { 8- Aspirin. }\end{array}$ & $\begin{array}{l}\sqrt{ } \\
\sqrt{ }\end{array}$ & $\mathrm{X}$ \\
\hline
\end{tabular}




\section{Results}

1- The unit's protocol was followed by staff in cardiology unit in $100 \%$ of cases.

2- A description of the characteristics of children admitted with DCM is presented in Figs. (1-5).

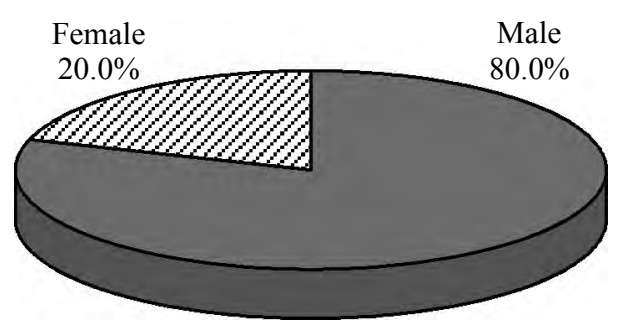

Fig. (1): Shows the prevalence of male \& female children admitted with DCM. The prevalence of male sex was $80 \% \& 20 \%$ of cases were females.

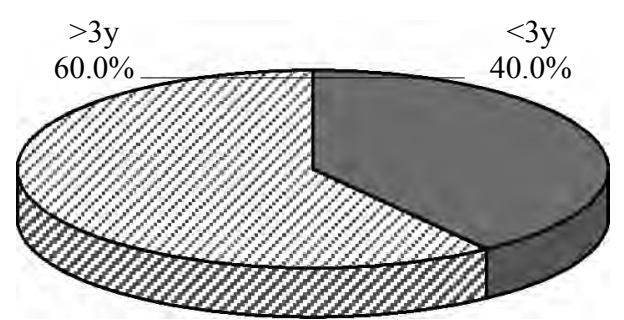

Fig. (2): Shows the prevalence of infants and children less than 3 year of age with DCM compared to older children. Cases $\leq 3$ years constituted $40 \%$ of cases. In $60 \%$ of cases the age was older than $3 y$ up to 16 years of age.

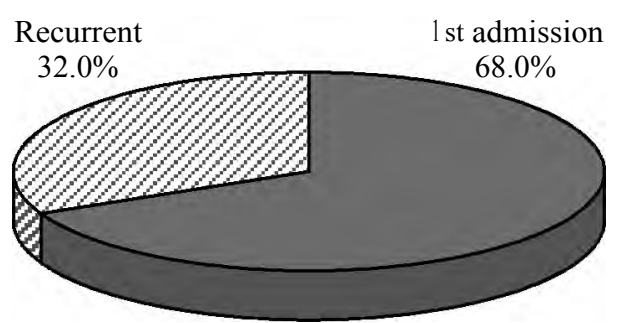

Fig. (3): Shows the prevalence of DCM cases presenting as first admission compared to those with recurrent admissions. The number of cases presenting as first admission was $68 \%$ \& in $32 \%$ of cases the admission was recurrent.

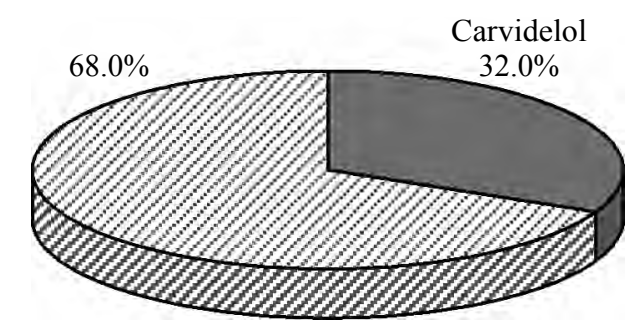

Fig. (4): Shows the prevalence of use of carvidelol in children with DCM. Carvidelol was used in $32 \%$ of children with DCM \& in the rest of cases (68\%) carvidelol was not used.

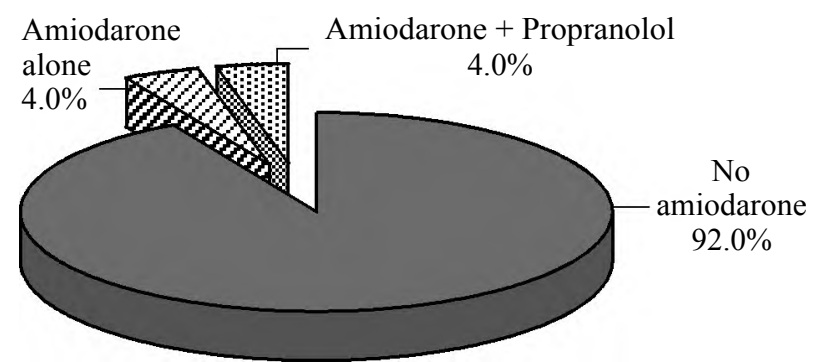

Fig. (5): Shows the prevalence of use of amiodarone (alone or in combination with propranolol) in children with DCM. Amiodarone was used in 2 cases $(8 \%$ of the cases): In one case it was used alone, and in the second case it was used in combination with propranolol.

\section{Discussion}

The difference between unit's protocol and the Canadian guidelines:

1- In the history: In the Canadian guidelines family history was obtained with a view of subsequent screening of family members for dilated cardiomyopathy as genetic basis for DCM was reported by Rubis, [12]. Only one third of children know the cause of their disease at time of diagnosis, while the remaining two third are classified as idiopathic (Fu et al., 2013) [6]. We recommend to add in the family history asking about sibling \& other members of the family with DCM and/or heart failure.

2- Regarding the investigations done, in the present study the CPK level was measured in order to differentiate cases that started as myocarditis from the rest cases with dilated cardiomyopathy. Although it was not stated in the Canadian guidelines, it was mentioned in other guidelines, as a help in laboratory differential diagnosis of myocarditis [13].

3- In cases of DCM presenting with arrhythmia, in this study amiodarone was the first line of treatment. Although this drug was not mentioned in the Canadian guidelines it was reported by Myung, [11] as being effective and relatively safe in children with DCM having tachyarrhythmia. Similarly in the present study the use of Propranolol as a second line antiarrhythmic drug in cases with no response to amiodarone was not mentioned in the Canadian guidelines. However, Myung, [11] reported beneficial effect of B-blocker (Propranolol) in children with DCM.

4- L-carnitine and Aspirin used by us in unit's protocol were also not mentioned in the Canadian guidelines but it was recommended by Myung, [11] as minimizing the risk of thromboembolic manifestation. 


\section{Conclusion and Recommendation:}

Regarding the adherence to unit's protocol this was present in $100 \%$ of cases. The difference between the present protocol and Canadian guidelines points out the lack of genetic studies performed by Assiut University Children Hospital in cases with DCM. In fact many of the cases with Idiopathic Dilated Cardiomyopathy (IDCM) in children are now thought to be due to genetic or familial factors.

- This study realized that family cardiac screening by Echocardiography for all patients who were newly diagnosed as DCM, (at least for both parents and young siblings) appears to be mandatory. Furthermore prophylactic use of L-carnitin in patient sibling may be recommended.

- We recommend family screening of infants and children with DCM together with good family history taking to be observed and added to the agreed upon unit's protocol. This is to be in preparation for genetic screening in a future study on DCM.

- A further recommendation that is suggested from this study is doing echocardiography in all cases presenting with heart failure not secondary to congenital cardiac anomaly or obvious cardiopulmonary affection.

\section{Acknowledgment:}

Thanks to all my dear staff members, colleagues and relatives of patients in Assiut Children Hospital for their continuous encouragement, cooperation and help.

Last but not least; I would like to thank my family for supporting me spiritually through my life.

\section{References}

1- American Heart Association: Pediatric Cardiomyopathy. Circulation, 112: 2061-75, 2015.

2- AZHAR A.S.: Pediatric idiopathic dilated cardiomyopathy: A single center experience. J. Nat. Sci. Biol. Med., 4 (1): 145-8, 2013.
3- BAUGHMAN K.L.: Diagnosis of myocarditis: Death of Dallas criteria. Circulation, Jan. 31, 113 (4): 593-5, 2006.

4- FELKER G.M., KERRY L.L., DAVID A.B., et al.: Diuretic Strategies in Patients with Acute Decompensated Heart Failure N. Engl. J. Med., 364 (9): 797-805. Mar. 2011.

5- FRANCONE M.: Role of Cardiac Magnetic Resonance in the Evaluation of Dilated Cardiomyopathy: Diagnostic Contribution and Prognostic Significance 10.1155/2014/ 365404. Feb., 2014.

6- FU LIJUN, MEIRONG H., et al.: Primary carnitine deficiency and cardiomyopathy: Korean Circulation Journal, 43 (12): 785-92, 2013.

7- GOSWAMI, et al.: Medscape reference, drugs, disease and procedure, chief editor Ooi HH, 2014.

8- HONG Y.M.: Cardiomyopathies in children. Korean Journal of Pediatrics, 56 (2): 52-9, 2016.

9- HUGHES S.E. and McKENNA W.J.: New insights into the pathology of inherited cardiomyopathy. Doi: 10.1136/ hrt.2004.040337. Heart, 91 (2): 257-64, 2005.

10- KANTOR P.F., LOUGHEED J., ADRIAN D., et al.: Presentation, Diagnosis, and Medical Management of Heart Failure in Children: Canadian Cardiovascular Society Guidelines. Canadian Journal of Cardiology, 29: 1535e1552, 2013.

11- MYUNG K. PARK: Park's Pediatric Cardiology for Practitioners. Sixth edition. ISBN: 978-0-323-16951-6 Copyright $\odot 2014$ by Saunders, an imprint of Elsevier Inc. Part 5 Acquired Heart Disease. 18. Primary Myocardial Disease, pp. 532-64, 2014.

12- RUBIS P.: The diagnostic work up of genetic and inflammatory dilated cardiomyopathy. The ESC Council for Cardiology Practice, Vol. 13, No. 19, 2015.

13- SNAB A.: Dilated cardiomyopathy: A disease of the myocardium. Biological \& Pharmaceutical Bulletin, 36: 18-22, 2013.

14- SCHANNWELL C.M., SCHOEBEL F.C., MARX R., et al.: Prognostic relevance of left ventricular diastolic function parameters in dilated cardiomyopathy. Z. Kardiol. April, 90 (4): 269-79, 2001

15- SPICER R.L. and WARE S.M.: Nelson textbook of pediatrics Edition 20, Section 6, Chapter 439: Diseases of the myocardium, p.p. 2271-8, 2016.

16- TOWBIN J.A., LOWE A.M., COLAN S.D., et al.: Incidence, causes, and outcomes of dilated cardiomyopathy in children. J.A.M.A., 296 (15): 1867-76, 2006. 


\section{علاج إعتلال عضلات القلب فى الآطفال بوحلدة قلب الآطفال

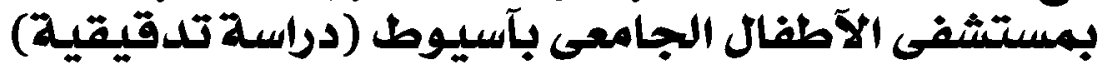

إن الهدف من هذه البحث هو : تقييم درجة إلتزام الوحدة بيرتوكول التعامل مع حالات إعتلال عضلات القلب التمددى ومقارنة بتوجيهات التعامل مع هذه الحالات بالإشادات المقبولة عالميا لذانلك.

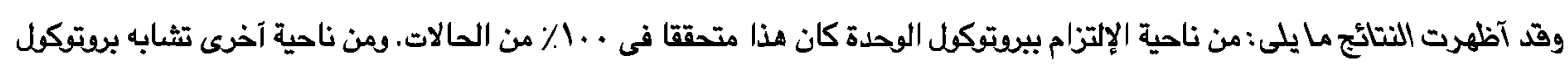

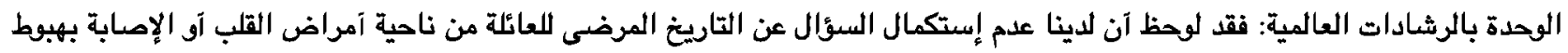

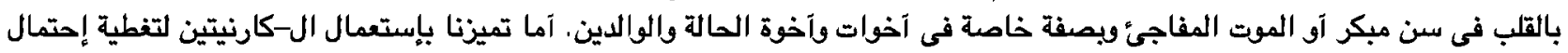

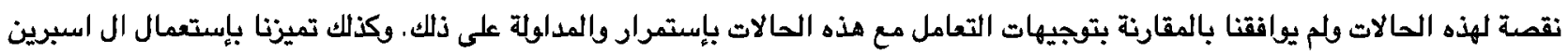

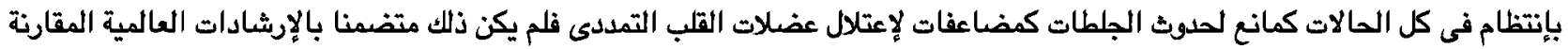

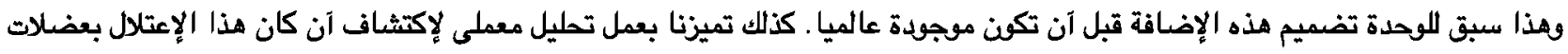

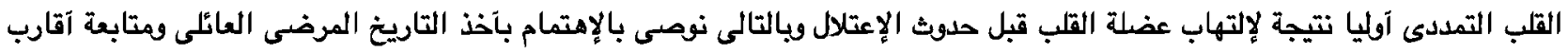

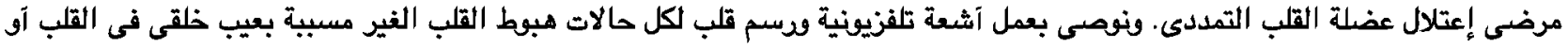
بسبب مرض فى الرئتين. 